\title{
SmART-ER imaging and treatment of glioblastoma
}

\author{
Patrick V. Granton ${ }^{1}$ Sanaz Yahyanejad ${ }^{1} \cdot$ Marc A. Vooijs $^{1}$ (I)
}

Received: 6 April 2015/Accepted: 23 April 2015/Published online: 8 May 2015

(C) Springer Science+Business Media New York 2015

To the Editor,

Small animal imaging and radio therapy (SmART) platforms combine non-invasive imaging and precise conformal irradiation and are emerging as an important links between preclinical cancer research and clinical treatment to test novel irradiation schedules, evaluate radiosensitizers, and to monitor disease progression and response [1].

With interest we read the article by Kirschner et al. "In vivo micro-CT imaging of untreated and irradiated orthotropic glioblastoma xenografts in mice: capabilities, limitations and a comparison with bioluminescence imaging" in Journal of Neuro-Oncology [2]. Kirschner has used a comparable approach as in Yahyanejad et al. "Complementary Use of Bioluminescence Imaging and ContrastEnhanced Micro-Computed Tomography in an Orthotropic Brain Tumor Model" Mol Imaging [3].

Both studies describe the implementation of SmART for a preclinical Glioblastoma (GBM) model in mice. GBM is a deadly disease with no cure and in dire need of new treatment options.

Both studies investigated the correlation between bioluminescent imaging (BLI) to contrast-enhanced micro-CT (CECT) as a tool to estimate tumor volume and monitor disease progression and response. BLI is a cheap, fast and very sensitive non-invasive based imaging approach based

Electronic supplementary material The online version of this article (doi:10.1007/s11060-015-1801-6) contains supplementary material, which is available to authorized users.

Marc A. Vooijs

marc.vooijs@maastrichtuniversity.nl

1 Department of Radiotherapy (MAASTRO)/GROW- School for Developmental Biology \& Oncology, Maastricht University, Maastricht, The Netherlands on the expression of the enzyme firefly luciferase in tumor cells, which emits lights when the substrate luciferin is injected. Micro-CT based follow up of brain tumor growth requires the use of exogenous contrast agents.

Both studies use the U87MG GBM cell line that establishes relatively quickly $(\sim 1$ month) so that therapeutic interventions can be performed in a reasonable timeframe. In Kirschner's study, immunosuppressed NOD/SCID mice were used, while in our study CD1 nude mice were used. Similar surgical techniques were used between the two studies, except that in our study fewer cells were injected $\left(1\right.$ vs. $\left.2.5 \times 10^{6}\right)$. In our study, all mice that were implanted $(100 \%, n=33)$, established tumors, whereas in the other study only three quarters grew tumors $(74 \%, n=27)$.

CECT imaging was used in both studies with similar energies $(80 \mathrm{kVp})$. Both studies used Iodinated contrast agents iomeprol $(300 \mu \mathrm{l} / \mathrm{iv})$ or Ominipaque $350(150 \mu \mathrm{l} / \mathrm{iv})$, which both appear to be well tolerated. The CECT-derived tumor volumes compared to histological determination were systematically smaller in both studies 23 and $20 \%$, respectively, most likely as a consequence of tissue fixation. Importantly, both studies observed strong correlation coefficients of 0.96 and 0.88 between micro-CT and histology demonstrating that CECT imaging in orthotropic GBM mouse model can be used to delineate brain tumor growth longitudinally in vivo (supplementary material). Additionally, both studies reported good inter- and intra-observer correspondence between $\mu \mathrm{CT}$ delineated tumor volumes. This is crucial because micro-CT could suffice for initial tumor delineation and planning and BLI imaging is accurate enough (across $5 \times$ tumor volume doublings) to measure tumor control in response to treatments.

To monitor tumor growth longitudinally both studies used U87MG tumor cells expressing firefly luciferase. While the Kirschner's study reported a moderate correlation (Spearman $\mathrm{r}=0.543$ ) between CT-derived tumor 
volume and BLI intensity, a strong correlation was demonstrated in our study (Spearman $r=0.819$ ). This might be explained by the fact that the Yahyanejad study used significantly more data points including tumor volumes greater than $10 \mathrm{~mm}^{3},(\sim$ sphere diameter $2.7 \mathrm{~mm})$ to demonstrate a correlation. They found that below $10 \mathrm{~mm}^{3}$ significant errors in the correlation between BLI and micro-CT were observed. Therefore, drawing meaningful correlations for volume sizes from BLI below this minimum size is not recommended.

In conclusion, both studies show the value of CECT to delineate and calculate tumor volumes in vivo and BLI as a surrogate marker for measuring tumor volumes.

In the near future we anticipate an accelerated development of image guided radiotherapy platforms such as SmART with additional on-board capabilities (e.g. ultrasound, BLI and MRI, PET). Important aspects like organ movement (4D) and normal tissue toxicity key challenges in the clinic today will be experimentally addressed and provide solutions that will advance the discovery of new interventions beneficial to patient outcome.
Conflict of interest The authors declare that they have no conflict of interest.

\section{References}

1. Verhaegen F, Granton PV, Tryggestad E (2011) Small animal radiotherapy research platforms. Phys Med Biol 56:55-83

2. Kirschner S, Felix MC, Hartmann L, Bierbaum M, Maros ME, Kerl HU, Wenz F, Glatting G, Kramer M, Giordano FA, Brockmann MA (2015) In vivo micro-CT imaging of untreated and irradiated orthotopic glioblastoma xenografts in mice: capabilities, limitations and a comparison with bioluminescence imaging. J Neurooncol 122:245-254

3. Yahyanejad S, Granton PV, Lieuwes NG, Gilmour L, Dubois L, Theys J, Chalmers AJ, Verhaegen F, Vooijs MA (2014) Complementary use of bioluminescence imaging and contrast-enhanced micro-computed tomography in an orthotopic brain tumor model. Mol Imaging 13:1-8 